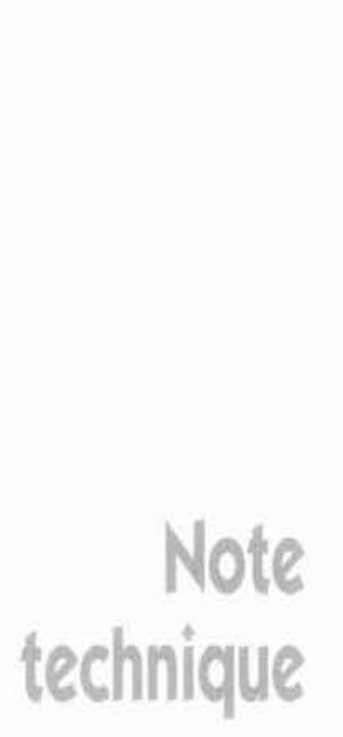

NDLR : Les discussions sur cet article sont acceptées jus. qu'au 1er février 2006.

\section{Le butonnage dans un coin pour une fouille ouverte d'un côté Angle buttress}

P. HABIB

LMS, Ecole polytechnique
Victor Hugo dans Les Misérables écrit: :Grâce à ses nombreuses évasions du bagne de Toulon, Jean Valjean était passé maître dans cet art incroyable de s'élever sans échelles, sans crampons, par la seule force musculaire, en s'appuyant de la nuque, des épaules, des hanches et des genoux, en s'aidant à peine des rares reliefs de la pierre, dans l'angle droit d'un mur, au besoin jusqu'à la hauteur d'un sixième

C'est très difficile de grimper dans un coin: il faut une adhérence terrible des chaussures au contact simultané des deux murs. Compte tenu du poids propre, il faut un coefficient de frottement largement supérieur à $45^{\circ}$. Faire

(1) Victor Hugo «Les Misérables》. Deuxième partie. Livre cinquième. Chapitre V: « Qui serait impossible avec l'éclairage au gaz.1, p. 634 dans l'édition 1998 des a Classiques de Poche $n$. Dans les pages suivantes, ce procédé sera utilisé par Jean Valjean pour êchapper aux auxiliaires du étage ${ }^{1} \cdot n$ policier Javert. de la varappe en grimpant dans une "cheminée» dont les parois lisses sont presque parallẻles, c'est plutôt facile mais, dans un coin à $90^{\circ}$, c'est presque impossible. Souvent, il y a une fissure au fond du coin. On peut alors monter à la « Düllfer »), mais la fissure n'est pas toujours accessible...

Venons-en au butonnage dans les coins d'une fouille pour le soutènement des murs ou des parois moulées. Si les fouilles sont carrées ou rectangulaires, des butons placés en coin sont un soutènement efficace (Fig. 1). Les butons ne dérapent pas quand ils sont fixés efficacement sur les murs et si les murs sont aptes à supporter la compression horizontale correspondante, La force transmise par un buton peut alors se décomposer sur chaque mur en une force normale qui s'oppose à la poussée des terres et une force tangentielle $\mathrm{T}$ qui est équilibrée par la force $\mathrm{T}$ engendrée par le buton du coin adjacent.

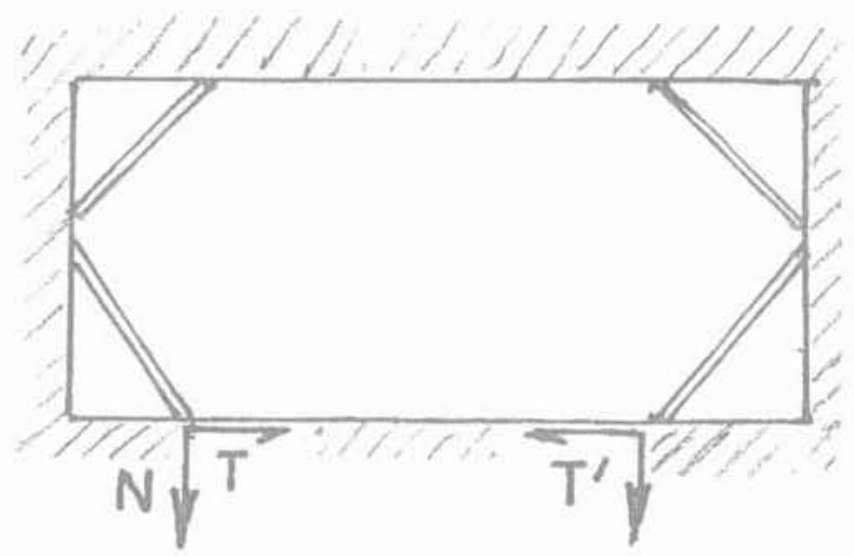

FG.1 Fouille rectangulaire.

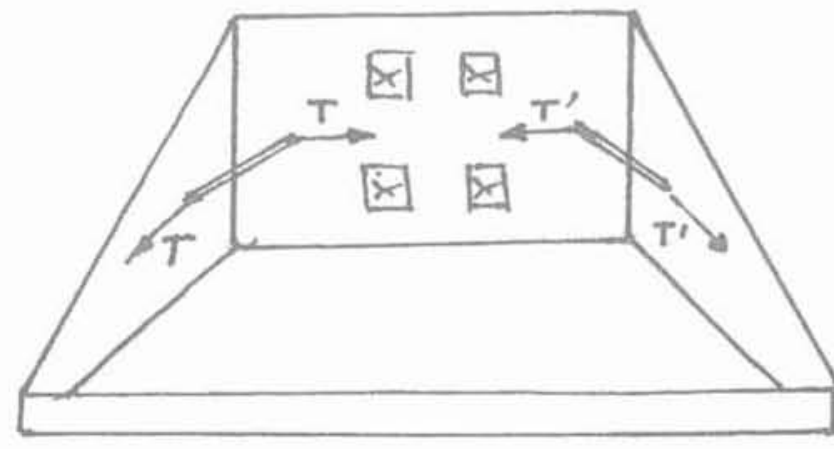

FIG. 2 Fouille dans un sol en pente. 


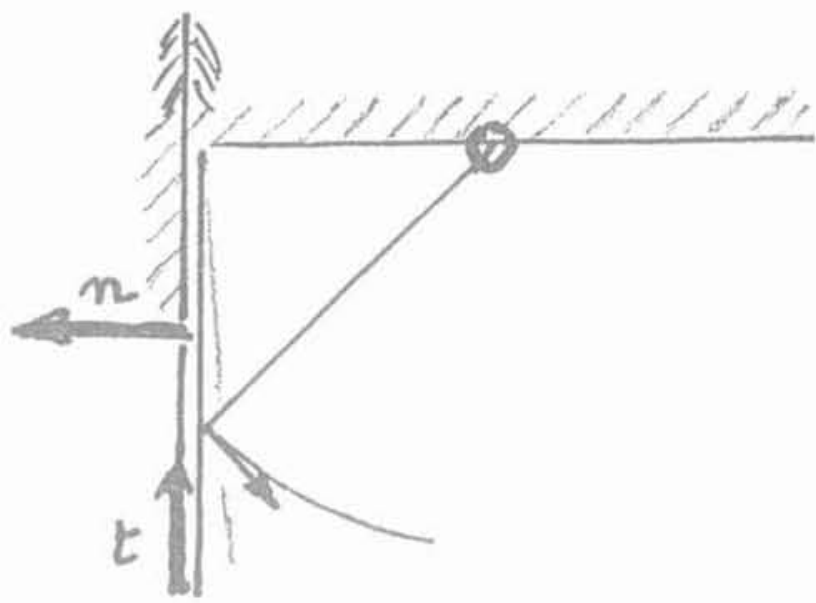

FG. 3. Cinématique du mouyement.

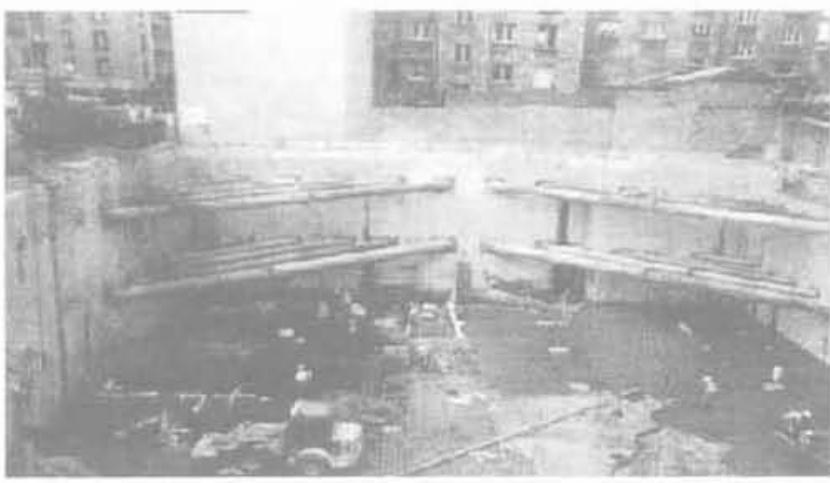

FIG. 4. Un exemple de butonnage d'un coin.

Mais, que se passe-t-il s'il n'y a pas de coin adjacent, par exemple parce que la fouille est ouverte d'un côté ou pour d'autres cas, comme lorsque le sol est en pente (Fig. 2)? Le côté du mur le plus haut de la fouille est évidemment soumis à la plus grande poussée des terres: souvent d'ailleurs ce mur est soutenu par des ancrages ou par l'encastrement de son pied. Les forces tangentielles sur ce mur, apportées par les butons, sont équilibrées. Mais, pour les murs latéraux ?

Le calcul est fait le plus souvent en déterminant alors la résistance du mur au glissement contre le sol par une formule de la forme $\mathrm{T}=\mathrm{S}(\mathrm{c}+\mathrm{n} \tan \varphi)$, oú $\mathrm{S}$ est I'aire du mur en contact avec le sol et n la poussée apportée par le buton. En somme, on vérifie deux fois l'équilibre du mur, d'abord par le calcul de la poussée des terres et ensuite par le non glissement du mur le long du sol. Ceci n'est pas correct, car le mécanisme de ruine, c'est la somme des deux. D'ailleurs, la cinétique du début de l'accident est claire et simple. Comme d'une part le raccourcissement d'un buton métallique est insignifiant, et que, d'autre part, une extrémité du buton est fixée sur un mur qui ne bouge pas, il en résulte que la trajectoire du point de contact de l'autre extrémité du buton sur le mur latéral est pratiquement un arc de cercle (Fig. 3). Ainsi, au début d'un incident le déplacement normal du mur latéral vers la fouille est accompagné d'un mouvement tangentiel du mur latéral de même amplitude dans le sens de la pente. Si le soutènement est constitué de plusieurs butons dans de mêmes plans horizontaux, comme sur la figure 4 , et pour peu que la paroi latérale prenne un peu de ventre, elle peut s'étirer dans la direction horizontale et donc se rompre avec des fissures verticales, la parol étant alors soumise à une traction horizontale. Alors, c'est la panique !

Et cette situation est tout à fait êtrange. En effet, lorsqu'un contrôleur passe sur le chantier avant tout désordre, et qu'il voit ce que montre la figure 4, il pense "Ah ! Oui ! C'est très bien: c'est évident, ça tient grâce à ces très beaux butons ». Mais, quand les déplacements apparaissent et que la catastrophe menace, le mème contrôleur venant sur le chantier dira: " Mais évidemment, comment voulez-vous que ces butons tiennent quelque chose? "y.

Mais alors, que peut-on faire ? On peut évidemment ajouter une force normale à la paroi, c'est-à-dire augmenter $n$ dans la formule donnée plus haut, de façon à faire croître le terme n tan $\varphi$. Pour cela on peut, par exemple, placer des tirants perpendiculaires au mur latéral. Mais alors, si on équilibre la poussée des terres par des ancrages, on n'a plus besoin de butons.

On peut aussi mettre en ceuvre des tirants longs horizontaux derrière le mur latéral, et parallèles à ce dernier, en nombre suffisant pour s'opposer à T (Fig. 3). Ce sera toujours plus coûteux que si on avait mis tout de suite des ancrages normaux aux murs latéraux.

En conclusion, le butonnage en coin pour une fouille ouverte d'un côté est une méthode dangereuse, ou bien si la terre ne pousse pas derrière le mur latéral, c'est une méthode illusoire.

Il reste néanmoins un problème qui peut paraître intéressant: si l'angle du coin est aigu, c'est-à-dire plus petit qu'un angle droit, jusqu'à quelle limite de cet angle le butonnage d'un seul coin peut-il être réalisé sans risques? 